\title{
The Nature and Role of Periosteum in Bone and Cartilage Regeneration
}

\author{
Seika Matsushima ${ }^{a-c}$ Noritaka Isogai $^{a, c}$ Robin Jacquet ${ }^{a, b}$ Elizabeth Lowder ${ }^{a, b}$ \\ Taku Tokui ${ }^{a, c}$ William J. Landis ${ }^{a}$, \\ a Department of Integrative Medical Sciences, Northeastern Ohio Universities Colleges of Medicine and Pharmacy, \\ Rootstown, Ohio, and ${ }^{b}$ Department of Polymer Science, The University of Akron, Akron, Ohio, USA; \\ 'Department of Plastic and Reconstructive Surgery, Kinki University Medical School, Osaka-sayama, Japan
}

\section{Key Words}

Periosteum $\cdot$ Bone $\cdot$ Cartilage $\cdot$ Regeneration $\cdot$

Tissue engineering

\begin{abstract}
This study was undertaken to determine whether periosteum from different bone sources in a donor results in the same formation of bone and cartilage. In this case, periosteum obtained from the cranium and mandible (examples of tissue supporting intramembranous ossification) and the radius and ilium (examples of tissues supporting endochondral ossification) of individual calves was used to produce tissue-engineered constructs that were implanted in nude mice and then retrieved after 10 and 20 weeks. Specimens were compared in terms of their osteogenic and chondrogenic potential by radiography, histology, and gene expression levels. By 10 weeks of implantation and more so by 20 weeks, constructs with cranial periosteum had developed to the greatest extent, followed in order by ilium, radius, and mandible periosteum. All constructs, particularly with cranial tissue although minimally with mandibular periosteum, had mineralized by 10 weeks on radiography and stained for proteoglycans with safranin-O red (cranial tissue most intensely and mandibular tissue least intensely). Gene expression of type I collagen, type II collagen, runx2, and bone sia-
\end{abstract}

\section{KARGER}

Fax +4161306 1234

E-Mail karger@karger.ch

www.karger.com (c) 2011 S. Karger AG, Basel

Accessible online at: www.karger.com/cto loprotein (BSP) was detectable on QRT-PCR for all specimens at 10 and 20 weeks. By 20 weeks, the relative gene levels were: type I collagen, ilium $>>$ radial $\geq$ cranial $\geq$ mandibular; type II collagen, radial $>$ ilium $>$ cranial $\geq$ mandibular; run $\times 2$, cranial $\gg>$ radial $>$ mandibular $\geq$ ilium; and BSP, ilium $\geq$ radial $>$ cranial $>$ mandibular. These data demonstrate that the osteogenic and chondrogenic capacity of the various constructs is not identical and depends on the periosteal source regardless of intramembranous or endochondral ossification. Based on these results, cranial and mandibular periosteal tissues appear to enhance bone formation most and least prominently, respectively. The appropriate periosteal choice for bone and cartilage tissue engineering and regeneration should be a function of its immediate application as well as other factors besides growth rate.

Copyright $\odot 2011$ S. Karger AG, Basel

\section{Abbreviations used in this paper \\ BSP bone sialoprotein \\ QRT-PCR quantitative reverse transcription-polymerase chain reaction}

Dr. William J. Landis

Department of Polymer Science

Goodyear Polymer Center, Room 1215

The University of Akron, Akron, OH 44325-3909 (USA)

Tel. +1 330972 8483, E-Mail wlandis@uakron.edu 


\section{Introduction}

The growth, development, and regeneration of bone and cartilage depend on the presence of periosteum, a thin strip of soft tissue enveloping the midshafts of long bones and extending to their distal and proximal metaphyses and adjacent epiphyses [Malizos and Papatheodorou, 2005]. Periosteum is known to be pluripotent, consisting of osteoblastic and chondrogenic cells, and it may be utilized to engineer new bone formation in vivo [Stevens et al., 2005]. These cell types are well characterized in terms of function, expression of genes, synthesis and secretion of cellular and extracellular proteins and other molecules, and structural organization specific for the elaboration of bone and cartilage, respectively. However, less well understood are the nature and role of periosteum and its composite cells in the bone and cartilage formative and repair processes. Indeed, a critical question is whether periosteum from different bone sources in a vertebrate elicits identical responses in producing new bone and cartilage.

In this context, there have been but a few reports investigating possible differences and effects on osteogenesis and chondrogenesis of periosteum from various tissue sources in the same animal. Of these, periosteum from only certain bones of various animals and at different ages was examined [O'Driscoll and Fitzsimmons, 2001; O'Driscoll et al., 2001; Fan et al., 2008]. Results of these and related studies [Kwon et al., 2002; Szulc et al., 2006], therefore, could not be easily correlated. On the other hand, an analysis of cartilage from auricular, articular, nasoseptal, and costal sources of single calves showed that each retained its own specific characteristics of gene expression, protein and proteoglycan secretion, growth, and development [Kusuhara et al., 2009]. In the present study, periosteal tissue samples were harvested from four sites in individual young calves, the cranium and mandible (which undergo intramembranous ossification) and radius and ilium (which undergo endochondral ossification). Periosteal specimens were fabricated into tissue-engineered constructs that were implanted in nude mice and then retrieved and compared in terms of osteogenic and chondrogenic potential by radiographic, histological, and gene expression analyses. The summary data demonstrate different osteogenic and chondrogenic effects on utilizing periosteum from various tissue sources in the same animal.

\section{Materials and Methods}

This study and all protocols and procedures related to the care and maintenance of animals were approved by the Institutional Animal Care and Use Committee of the Northeastern Ohio Universities Colleges of Medicine and Pharmacy. Fresh tissues from 1- to 6-month-old calves were obtained from a local slaughterhouse, immediately placed on ice, and returned to the laboratory. Intact periosteal strips $(\sim 1 \times 1 \times 0.2 \mathrm{~cm}$ in length, width, and thickness, respectively) from the cranium, mandible, radius, and ilium were dissected from the same animal under sterile surgical techniques, and they were then sutured ( $n=3$ for each tissue source) with biodegradable 5-0 vicryl thread (Ethicon, Somerville, N.J., USA) to small scaffold blocks $(\sim 1 \times 1 \times 0.5 \mathrm{~cm})$ of hydroxyapatite-poly(L-lactide- $\varepsilon$-caprolactone) [HA-P(LA-CL); Gunze Co., Kyoto, Japan]. Scaffolds without periosteum were used as controls $(n=3)$. Cranial periosteum was consistently obtained from the frontal region of calf skulls as opposed to potentially different periosteum from other regions. The periosteum/scaffold constructs and counterpart controls were then incubated $\left(37^{\circ} \mathrm{C}\right.$, $5 \% \mathrm{CO}_{2}$ ) for $\sim 1$ week in separate petri dishes containing M199 medium supplemented with $10 \%$ fetal bovine serum and $1 \%$ antibiotic/antimycotic [Wada et al., 2009]. After incubation, constructs and controls were implanted in the dorsal subcutaneous space of 4- to 6-week-old male athymic (nu/nu) mice (Harlan Laboratories, Indianapolis, Ind., USA) for 10 and 20 weeks [Isogai et al., 1999, 2006; Landis et al., 2005; Wada et al., 2009]. Individual mice carried only one implant. Following implantation, animals were sacrificed by $\mathrm{CO}_{2}$ overdose and the constructs or scaffold controls were surgically harvested. Characterization of specimens included gross observation and photography, X-ray radiography, histology, and measurement of gene expression by quantitative reverse transcription-polymerase chain reaction (QRT-PCR) analysis.

Upon their retrieval, constructs were quickly bisected longitudinally. Half of each specimen was fixed in $10 \%$ neutral buffered formalin for $24 \mathrm{~h}$, examined and documented by X-ray radiography [Wada et al., 2009], and then dehydrated in graded ethanols, embedded in paraffin, and processed for histological study. Samples were cut on a microtome into 5 - $\mu$ m-thick sections and then stained in various solutions. The latter included toluidine blue to reveal the general morphology of the specimens, safranin-O red for the detection of secreted proteoglycans by periosteal chondrocytes, a von Kossa solution to demonstrate phosphate, and alizarin red to show calcium. Mineral deposits of calcium phosphate were determined by merging recorded images of serial sections stained separately with von Kossa or alizarin red; overlapping stained sites were those mineralized and correlated with X-ray radiography.

The remaining half of each retrieved construct was immersed in RNAlater ${ }^{\circledR}$ (Ambion/Life Technologies, Carlsbad, Calif., USA), frozen in liquid nitrogen, and stored at $-80^{\circ} \mathrm{C}$ for subsequent QRT-PCR analysis [Jacquet et al., 2005; Wada et al., 2009]. The genes examined were related to bovine bone and cartilage formation and mineralization and included type I collagen, type II collagen, runx2, and bone sialoprotein (BSP). Specimens were ground to powder under liquid nitrogen in a Spex mill (Model 6750; Spex, Inc., Metuchan, N.J., USA) and total RNA was isolated and reverse-transcribed [Jacquet et al., 2005]. Bovine-specific primers for QRT-PCR analysis of the above mentioned genes and cyclophilin D as a normalizing gene were designed. An ABI 
Fig. 1. Gross morphology and corresponding X-ray radiographs of intact tissue-engineered constructs composed of scaffold and periosteum from different sources in the same $\sim 5$-month-old calf. After 20 weeks of implantation, tissue development varied among the constructs (a) as did the presence of mineral deposits (b). Scaffolds alone contained mineral as hydroxyapatite and contributed intrinsic X-ray opacity (relatively bright image regions). Scale bar $=5 \mathrm{~mm}$ (a, b).

Fig. 2. Representative thick (5 $\mu \mathrm{m})$ sections from $\sim 5$-month-old calf constructs retrieved after 10 (a) or 20 (b) weeks of implantation in nude mice. Sections in $\mathbf{b}$ were obtained from the samples in figure la. Two examples of sections from control scaffolds without sutured periosteum are also shown. Sections were treated with von Kossa and safranin-O red solutions for the detection of phosphate and proteoglycans, respectively. Over the two time intervals for implantation, the size or extent of development differed among the constructs sutured with periosteum, as well as the presence and distribution of phosphate (as a surrogate for mineral deposition) and proteoglycans. Section enlargements of 20 -week implants show details of structure and staining (c). $\mathrm{S}=$ Scaffolds; $\mathrm{C}=$ cartilage proteoglycans; $\mathrm{M}=$ mineral; $\mathrm{P}=$ periosteum. Scale bars $=5 \mathrm{~mm}(\mathbf{a}, \mathbf{b})$ and $1 \mathrm{~mm}(\mathbf{c})$.

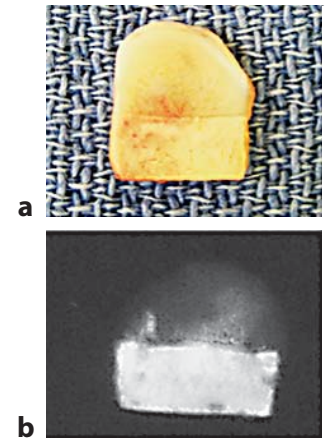

Cranium
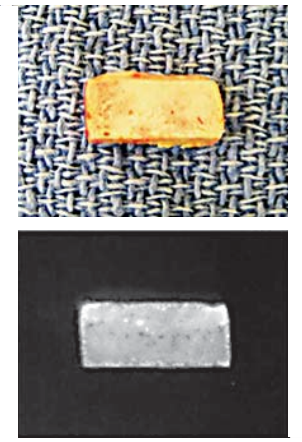

Mandible
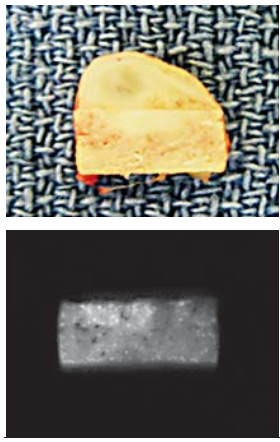

Radius
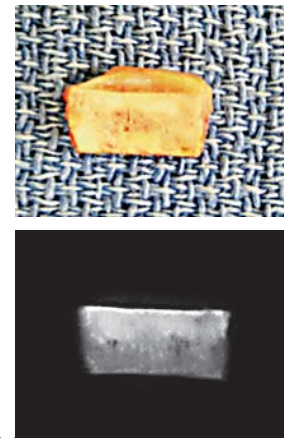

Ilium

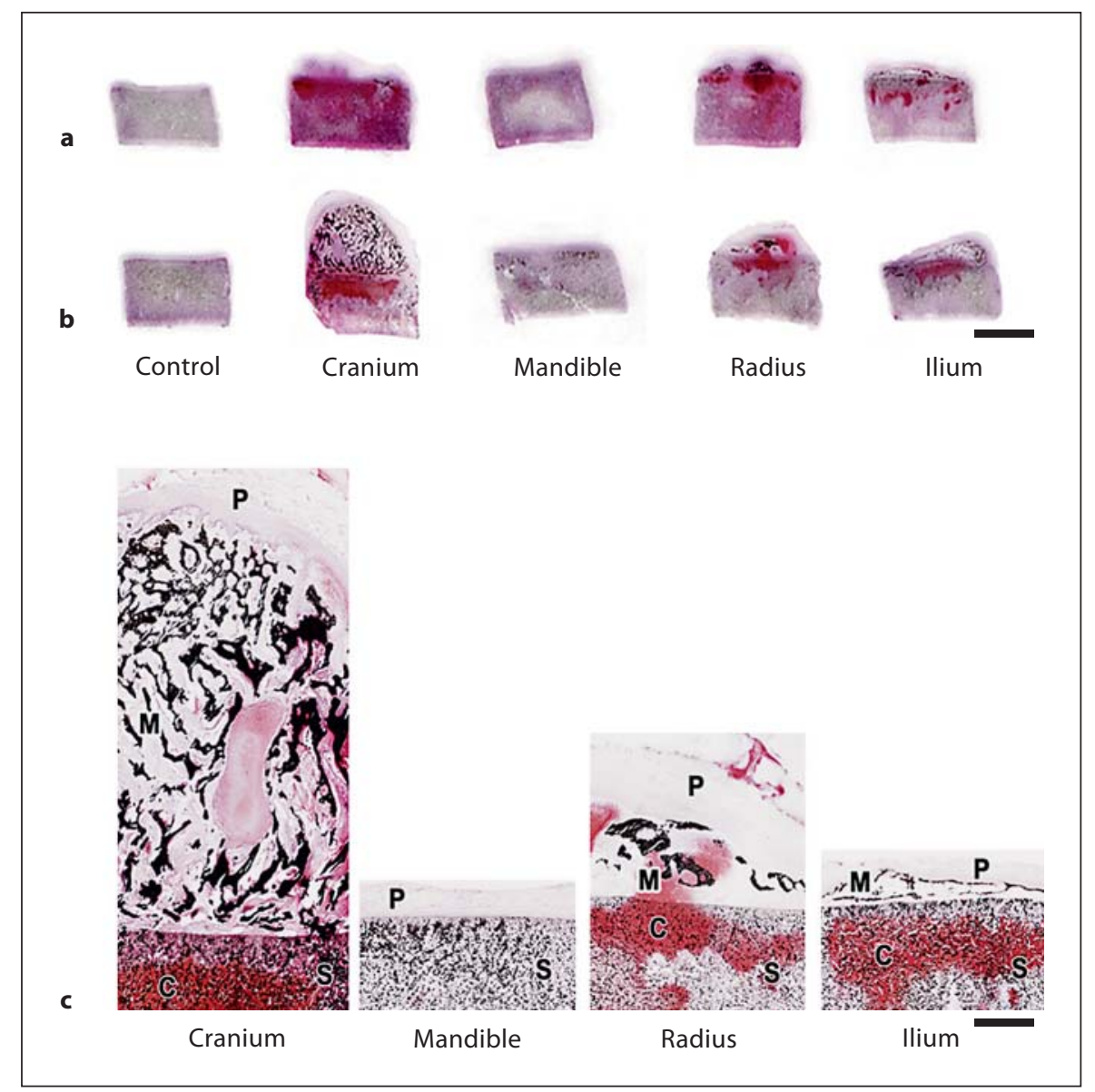

Prism 7500 Fast Sequence Detector (Applied Biosystems/Life Technologies, Carlsbad, Calif., USA) was used for quantitative PCR. Analyses followed the relative standard curve methodology outlined in Applied Biosystems User Bulletin \# 2 [Jacquet et al., 2005; PE Applied Biosystems, 1997]. Statistical analyses of gene expression from the various retrieved constructs were performed using ANOVA and a post hoc Bonferroni multiple comparisons test.

\section{Results}

Figure 1 shows the gross morphology and corresponding X-ray radiographs from tissue-engineered constructs consisting of HA-P(LA-CL) and periosteum obtained from the same $\sim 5$-month-old calf. The cell numbers per square micrometer of tissue for the four different sources 
of periosteum utilized in construct formation were calculated and found to be statistically equivalent. All constructs were harvested after 20 weeks of implantation in nude mice. Morphological differences among constructs were apparent, and at this harvest time constructs with cranial periosteum developed to the greatest extent, followed in order by radial, ilium, and mandibular periosteum. Mineralization detected on X-ray radiography also differed among constructs with the degree of mineral deposition following the order of periosteal development.

Figure 2 illustrates representative histological and gene expression data from periosteal/scaffold constructs developed from the same animal as noted in figure 1. Counterpart control scaffolds without tissue are also shown. The appearance of sections of the implants and scaffolds retrieved after 10 and 20 weeks of implantation in individual nude mice is shown in figure $2 \mathrm{a}, \mathrm{b}$, respectively. As in the gross specimens shown in figure 1a, growth and development varied for the implanted constructs but not for controls without tissue. Sections of constructs harvested at 10 weeks of implantation (fig. 2a) support the observations of sections from 20 -week harvested samples (fig. 2b) and 20-week gross morphology (fig. 1a) that cranial periosteum constructs exceeded their counterparts in development while mandibular periosteum constructs were least advanced. On von Kossa staining for phosphate to indicate mineral [with alizarin red staining for calcium (not shown)], all periosteal constructs, particularly with cranial tissue although minimally with mandibular periosteum, contained mineral deposits by 10 weeks and these increased by 20 weeks of implantation (fig. 2a, b). X-ray radiography (fig. 1b) yielded correlative results.

Figure $2 c$ shows enlargements of other sections from the 20-week harvested constructs presented in figures 1 and $2 \mathrm{~b}$. While the HA-P(LA-CL) scaffolds themselves were comprised in part of apatite, mineralization of the respective constructs was found principally in those regions that had developed within the periosteal tissue initially sutured to the scaffolds (fig. 2c). Mineral deposition took the form of evident trabeculae in the various constructs and most notably in the numerous seams of new mineral deposits appearing in a form resembling darkly staining, interconnected spicules in the constructs fabricated with cranial periosteum (fig. 2c). Similar darkly staining, punctuate deposits marked the developing tissues of ilium and radial periosteum sutured to and overlying the scaffolds (fig. 2c). Only in mandibular periosteal/scaffold constructs and the scaffold controls was mineral deposition difficult to detect aside

Periosteum in Bone and Cartilage

Regeneration from the apatite that was intrinsic to the scaffolds alone (fig. 2b, c).

On staining for proteoglycans with safranin-O red, cranial tissue was the most intensely reactive tissue and mandibular tissue the least (fig. $2 b, c)$. In this regard, the stain was observed both in the newly developing tissue originally sutured to the scaffolds and within the scaffolds themselves, particularly in constructs sutured with periosteum from the bovine cranium, ilium, and radius (fig. 2b, c). Control scaffolds without sutured periosteum and therefore devoid of cells were not stained with safranin-O red (fig. 2a, b).

Gene expression of type I collagen, type II collagen, runx2, and BSP was detectable by QRT-PCR for all periosteal-wrapped constructs following their implantation for both 10 and 20 weeks (fig. 3a-d). Each of the specimens developed from bovine periosteal tissues - cranium, mandible, radius, and ilium - was different in its quantitative expression levels on a per cell basis for these four genes of interest at both implantation times. Greater differences among the genes were generally found by 20 compared to 10 weeks of implantation (fig. 3a-d). At the longer implantation time of 20 weeks, the relative gene level summaries were: type I collagen, ilium $>>$ radial $\geq$ cranial $\geq$ mandibular; type II collagen, radial $>$ ilium $>$ cranial $\geq$ mandibular; runx2, cranial $\gg>$ radial $>$ mandibular $\geq$ ilium; and BSP, ilium $\geq$ radial $>$ cranial $>$ mandibular $\left({ }^{*} \mathrm{p}<0.1\right.$ for all genes by ANOVA and a post hoc Bonferroni multiple comparisons test).

\section{Discussion}

Data from this study show that each bovine cell-based construct, compared to the others and to scaffold controls without sutured periosteum, grows, develops, and mineralizes at its own rate over either 10 or 20 weeks of implantation in nude mice. Thus, the osteogenic and chondrogenic capacity of the pluripotent periosteum comprising the different constructs is not identical and varies depending on the periosteal tissue source regardless of intramembranous or endochondral processes of ossification. Cranial (intramembranous bone) and mandibular (intramembranous bone) periosteal tissues appear by histology and radiology to enhance bone, cartilage, and mineral formation most and least prominently, respectively, while radial and ilium (endochondral bones) periosteal tissues fall between their counterparts in inducing new tissue growth, development, and mineralization. Although variable in the constructs, cells, extracel- 


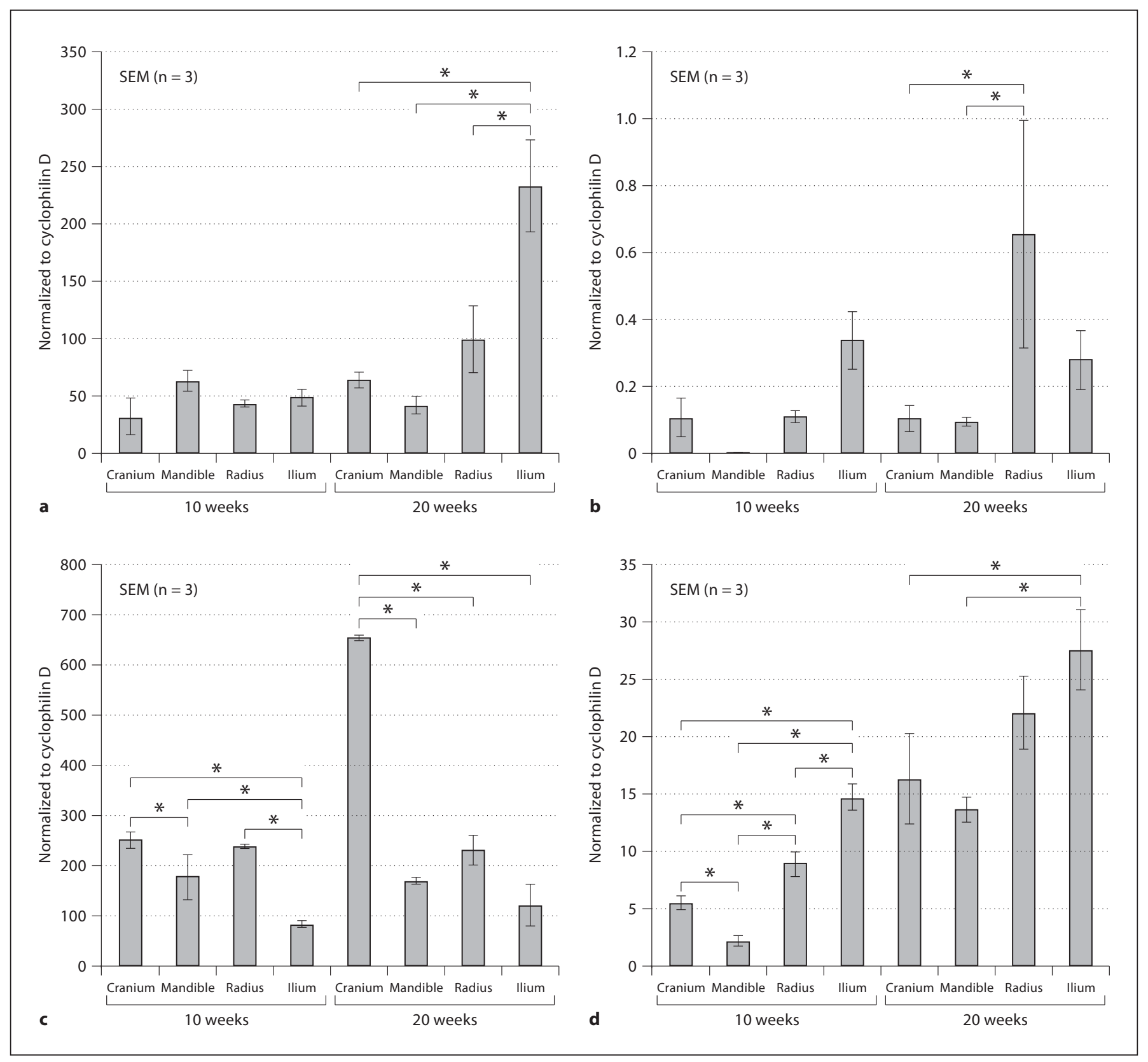

Fig. 3. QRT-PCR analyses of type I collagen (a), type II collagen (b), runx2 (c), and BSP (d) from frozen-ground specimens retrieved from nude mice after 10 and 20 weeks of implantation. Statistically significant differences $(\mathrm{p}<0.1)$ are designated by an asterisk $\left(^{*}\right)$ between given gene expression levels for the different constructs sutured with periosteum. Forward and reverse primers for bovine-specific type II collagen and BSP have been published [Kusuhara et al., 2009]. Bovine-specific primers for runx2, type I collagen, and cyclophilin D are as follows:

\begin{tabular}{lll}
\hline \multirow{2}{*}{ Runx2 } & F1781 & CCACTCTGTCACGATCTTGCAA \\
& R1866 & TGAAACCTGGTTAGGACTGTCTCTCT \\
\hline Type I $[\alpha 2(\mathrm{I})]$ collagen & F4299 & CATTTCTTCTGCACATCTACTTGCTT \\
& R4423 & AAATTCCAAACTTTTGGAGAGGG \\
\hline Cyclophilin D & F33 & GTTCTTCGCGGACCTTGTGA \\
& R106 & CGTGTATGACCGCCTCGTAA \\
\hline
\end{tabular}


lular matrices, and mineral appear within both the newly developing tissue overlying the construct scaffolds and the scaffolds themselves. These results indicate that osteoblasts and chondrocytes derived from sutured periosteum remain viable during implantation and migrate into the constructs. The cells proliferate and secrete matrix that leads to new bone and mineral formation (osteoblasts) and new cartilage (chondrocytes) in interior spaces of the scaffolds as well as in the tissue over the scaffolds. As anticipated, scaffold controls (without sutured periosteum) were not enhanced following staining or radiography and had no gene amplification. These results indicate that the tissue growth, development, and mineral formation of specimens failed to occur in the absence of periosteal cells.

After 20 weeks of implantation, the observed prominent regeneration of constructs comprised of cranial periosteum was consistent with very high runx 2 and elevated BSP gene expression levels, while the more slowly developing constructs consisting of mandibular periosteum were supported by only modest expression levels of all of the genes analyzed. These quantitative data were normalized and therefore represent expression values calculated per cell. At 20 weeks of implantation, higher levels of expression of type I collagen and BSP in con- structs with radial and ilium periosteum may reflect endochondral events in these tissues compared to the intramembranous activity of constructs with cranial and mandibular periosteum. Overall differences in type II collagen levels in all constructs were marginal considering the absolute normalization values of gene expression. Based on the present data and like other cartilage results [Kusuhara et al., 2009], the appropriate choice of periosteum to be utilized for bone and cartilage tissue engineering and regeneration should be a function of the specific bone intended to be augmented, and it should be determined by several other factors including expression levels of critical genes, protein or proteoglycan matrix production, and periosteal growth rate leading to osteogenic and chondrogenic capacity.

\section{Acknowledgments}

The authors thank the members of the Comparative Medicine Unit of the Northeastern Ohio Universities Colleges of Medicine and Pharmacy for their assistance in animal care and the use of facilities. This study was supported by grant AR41452 from the National Institutes of Health, Washington, D.C., USA (to W.J.L.), and by a Hitech Research Center grant from the Ministry of Education, Culture, Sports, Science, and Technology, Japan (to N.I.).

\section{References}

Fan, W., R. Crawford, Y. Xiao (2008) Structural and cellular differences between metaphyseal and diaphyseal periosteum in different aged rats. Bone 42: 81-89.

Isogai, N., W.J. Landis, T.H. Kim, L.C. Gerstenfeld, J. Upton, J.P. Vacanti (1999) Formation of phalanges and small joints by tissue engineering. J Bone Joint Surg Am 81: 306-316.

Isogai, N., H. Kusuhara, Y. Ikada, H. Otani, R. Jacquet, J. Hillyer, E. Lowder, W.J. Landis (2006) Comparison of different chondrocytes for use in tissue engineering of cartilage model structures. Tissue Eng 12: 691-703.

-Jacquet, R., J. Hillyer, W.J. Landis (2005) Analysis of connective tissues by laser capture microdissection and reverse transcriptasepolymerase chain reaction. Anal Biochem 337: 22-34.

Kusuhara, H., N. Isogai, M. Enjo, H. Otani, Y. Ikada, R. Jacquet, E. Lowder, W.J. Landis (2009) Tissue engineering a model for the human ear: assessment of size, shape, morphology, and gene expression following seeding of different chondrocytes. Wound Repair Regen 17: 136-146.
Kwon, D.S., M.R. Spevak, K. Fletcher, P.K. Kleinman (2002) Physiologic subperiosteal new bone formation: prevalence, distribution, and thickness in neonates and infants. Am J Roentgenol 179: 985-988.

Landis, W.J., R. Jacquet, J. Hillyer, E. Lowder, A. Yanke, L. Siperko, S. Asamura, H. Kusuhara, M. Enjo, S. Chubinskaya, K. Potter, N. Isogai (2005) Design and assessment of a tissue-engineered model of human phalanges and a small joint. Orthod Craniofac Res 8: 303312.

Malizos, K.N., L.K. Papatheodorou (2005) The healing potential of the periosteum: molecular aspects. Injury 365: 513-519.

O’Driscoll, S.W., J.S. Fitzsimmons (2001) The role of periosteum in cartilage repair: review. Clin Orthop Relat Res 391(suppl): S190S207.
O’Driscoll, S.W., D.B. Saris, Y. Ito, J.S. Fitzsimmons (2001) The chondrogenic potential of periosteum decreases with age. J Orthop Res 19: 95-103.

PE Applied Biosystems (1997) User Bulletin \# 2: ABI PRISM 7700 Sequence Detection System, Foster City, Calif.

Stevens, M.M., R.P. Marini, D. Schaefer, J. Aronson, R. Langer, V.P. Shastri (2005) In vivo engineering of organs: the bone bioreactor. Proc Natl Acad Sci USA 102: 11450-11455.

-Szulc, P., E. Seeman, F. Duboeuf, E. Sornay-Rendu, P.D. Delmas (2006) Bone fragility: failure of periosteal apposition to compensate for increased endocortical resorption in postmenopausal women. J Bone Miner Res 21: 1856-1863.

Wada, Y., M. Enjo, N. Isogai, R. Jacquet, E. Lowder, W.J. Landis (2009) Development of bone and cartilage in tissue-engineered human middle phalanx models. Tissue Eng 15: 3765-3778. 